\title{
CONTRIBUTION DE LA BIOPSIE STEREOTAXIQUE DANS LA PRISE EN CHARGE DES TUMEURS CEREBRALES: A PROPOS DE 283 CAS
}

\section{CONTRIBUTION OF STEREOTAXIC BIOPSY IN THE MANAGEMENT OF BRAIN TUMORS: A PROPOS OF 283 CASES}

\author{
OUDRHIRI Mohammed Yassaad ${ }^{1}$ \\ LAGHMARI Mehdi ${ }^{2}$ \\ HEMAMA Mustapha ${ }^{1}$ \\ GANA Rachid ${ }^{1}$ \\ MAAQILI Moulay Rachid ${ }^{1}$ \\ BELLAKHDAR Fouad ${ }^{1}$
}

1. Service de Neurochirurgie, Hôpital Ibn Sina, Rabat, Maroc

2. Service de Neurochirurgie, CHU Mohammed VI, Université Cadi Ayyad, Marrakech, Maroc

E-Mail Contact - OUDRHIRI Mohammed Yassaad : yassaad1 (at) msn (dot) com

Mots-clés: Biopsie stéréotaxique, tumeur cérébrale, pinéal

Key words: Stereotaxic biopsy, brain tumors, pineal

\section{RESUME}

\section{Introduction}

L'avènement de la biopsie stéréotaxique (BST) et le développement du scanner et de l'IRM ont amélioré la prise en charge des tumeurs cérébrales. Le but de cette étude est d'évaluer le rôle de la BST dans la prise en charge des tumeurs cérébrales.

\section{Matériel et Méthodes}

Etude rétrospective de 1995 à 2008 concernant 283 patients consécutifs ayant des tumeurs cérébrales, ayant bénéficié d'une BST, colligés au service de Neurochirurgie. L'âge moyen était de 45.5 ans (9 à 84). La BST fut réalisée sous guidage scannographique dans 260 cas et sous guidage IRM dans 23 cas. Pour les tumeurs pinéales, depuis Janvier 2000, une artériographie avec temps phlébographique et IRM préopératoire ont été jugées utiles pour éviter les complications hémorragiques.

\section{Resultats}

Dans 271 cas, la BST était concluante dès la première tentative (95.7\%). La répétition de la BST a permis de rétablir un diagnostic précis. II s'agissait de lésions malignes (70\% des cas). La mortalité péri-opératoire est passé de $5,9 \%$ avant 2000 à $0 \%$ après 2000 (Chi-carré; $p=0,013$ ).

\section{Conclusion}

Les BST doivent être étagées et répétées en cas d'histologie non concluante. Pour les petites lésions profondes la BST en conditions IRM est nécessaire. Pour les tumeurs pinéales, elle doit être réalisée par voie orthogonale trans-temporale.

\section{ABSTRACT}

\section{Introduction}

Development of STB in association with CTscan and MRI improved the management of brain tumors. The aim of this study is to assess the real place of STB in the management of these tumors.

\section{Material and methods}

Retrospective study from 1995 to 2008 about 283 consecutive patients harbouring brain tumors collected in department of Neurosurgery. Mean age was 45.5 years (9 to 84). STB was performed under CT scan guidance in 260 cases and under MRI in 23 cases. Concerning pineal tumors, since January 2000 , angiography with phlebographic time are mandatory in order to avoid haemorrhage. 


\section{Results}

In 271 cases, STB was conclusive since the first attempt (95.7\%). Repetition of STB enabled to reach diagnosis when histology wasn't conclusive. Malignant tumors were encountered in $70 \%$ of the whole series. Mortality decreased from $5.9 \%$ before 2000 to $0 \%$ after 2000 (Chi-square; $p=0,013$ ).

\section{Conclusion}

STB must be staged and repeated when histology isn't conclusive. For small deeper lesions STB under MRI is mandatory. For pineal tumors, STB must be performed through an orthogonal transtemporal way.

\section{INTRODUCTION}

L'avènement de la biopsie stéréotaxique (BST) avec le développement du scanner et de l'IRM a permis une amélioration de la prise en charge des tumeurs cérébrales. L'évolution constante de cette procédure et la modification de la stratégie thérapeutique ont aboutit non seulement à la réduction des complications et de la mortalité, mais aussi à la limitation du taux de biopsies blanches. Le but de cette étude est d'évaluer le rôle de la BST dans la prise en charge des tumeurs cérébrales.

\section{MATERIEL ET METHODE}

II s'agit d'une étude rétrospective de 1995 à 2008 concernant 283 patients consécutifs, colligés au service de Neurochirurgie de l'hôpital Ibn Sina pour des tumeurs cérébrales, ayant bénéficié d'une BST. L'âge moyen était de 45.5 ans (de 9 à 84 ans). $22 \%$ avaient plus de 60 ans. $47 \%$ des patients étaient des femmes et $53 \%$ étaient des hommes. Tous les patients recrutés ont bénéficié d'une TDM cérébrale, et 201 parmi eux, ont bénéficié d'une IRM (71\%).

\section{La procédure chirurgicale}

281 patients ont bénéficié d'une BST sous anesthésie locale, et deux patients sous anesthésie générale.

\section{Cadres stéréotaxiques}

Depuis 1995, un cadre de type Komaiß compatible au scanner (TDM) a été utilisé, et depuis 2001 un cadre Radionics® TDM et IRM compatible est utilisé.

La procédure stéréotaxique peut être réalisée sous anesthésie locale (patients de plus de 60 ans ou présentant un risque anesthésique) ou sous anesthésie générale classique. La fixation du cadre stéréotaxique est simple ; elle est réalisée par l'intermédiaire de quatre pointes qui s'appuient, à travers la peau, sur la table externe de la voûte du crâne. Le cadre stéréotaxique est lui-même fixé à la table du scanner. Le repérage de la lésion est effectué directement sur la console de visualisation du scanner. Les coordonnées de hauteur (Z), de position dans le sens antéropostérieur (coordonnée $Y$ ) et de latéralité (coordonnée $X$ ) par rapport au centre géométrique du cadre sont relevées de façon très simple. Une fois que le cadre de stéréotaxie a été fixé sur la tête du patient, la localisation tumorale a nécessité un repérage par TDM dans 260 cas et par IRM dans 23 autres cas. La cible, la trajectoire ainsi que la zone de prélèvement étagé sont calculés avec précision, à l'aide d'un logiciel informatique adapté. La BST est en suite réalisée en salle opératoire. A travers une trépanation, la sonde est insérée jusqu'à la cible. Le nombre de fragments réalisés était en moyenne de 5,7 fragments (1 à 8) (figure 1 et 2).

Pour les tumeurs pinéales, depuis Janvier 2000, une étude artériographique avec des temps phlébographiques et une IRM préopératoire ont été jugées utiles afin de réduire le risque hémorragique.

Parallèlement, un certain nombre de gestes stéréotaxiques non biopsiques comportant notamment des repérages stéréotaxiques per-opératoires ou des évacuations de kystes ont été réalisés. Ces techniques ne font pas l'objet de cet article. 


\section{RESULTAT}

Les données cliniques ont été étudiées (table 1). Les céphalées étaient le maître symptôme retrouvé dans $73.5 \%$ des cas (208/283). Un déficit neurologique a été retrouvé dans $56 \%$ des cas (158/283). 18\% des patients ont présenté une comitialité (51/283). La baisse de l'acuité visuelle était retrouvée chez $14 \%$ des patients (39/283). La taille moyenne des processus mesurée sur IRM ou, au cas échéant sur TDM, était de $37.5 \mathrm{~mm}$ en moyenne avec des extrêmes variant de 9 à 100 $\mathrm{mm}$.

\section{Localisation tumorale}

L'analyse du site des tumeurs a mis en évidence que dans $74 \%$ des cas, la BST a été réalisée pour des lésions profondes (région pinéale, corps calleux, Ille ventricule). Et dans $26 \%$ des cas, pour des lésions situées en zone hautement fonctionnelle (aires du langage, capsule interne, région rolandique, insula...) (tableau 1).

\section{Résultats histologiques}

Dans 261 cas, l'échantillonnage a permis un examen anatomopathologique concluant dès la première tentative $(92 \%)$.

Dans 22 cas, la BST a dû être renouvellée au moins une fois avant d'obtenir une étude histologique concluante. Dans 16 cas, deux procédures de BST étaient réalisées et dans 6 cas, trois procédures étaient nécessaires. Les différents diagnostics histologiques établis après biopsie stéréotaxique (283 cas) sont représentés sur la figure 3. Dans la majorité des cas il s'agissait de lésions malignes ( $70 \%$ des cas), le plus souvent de gliomes malins ( $49 \%$ des cas).

\section{Fiabilité de la biopsie}

La biopsie s'est avérée fiable dans 261 cas (92\%). Dans 22 cas, le résultat s'est avéré imprécis le doute diagnostique restait trop important, la BST devait être répétée au moins une fois avant d'obtenir une étude histologique concluante. Une deuxième biopsie a été réalisée dans 16 cas. Dans 6 cas, 3 BST étaient nécessaires. La répétition d'une biopsie a dans tous les cas permis de rétablir un diagnostic précis. Le caractère étagé ou non et le nombre des prélèvements apparaissent comme des facteurs évidents de fiabilité de la biopsie.

\section{Complications de la BST}

Au cours de la première période 1995-2000, 7 complications hémorragiques sont survenues, parmi lesquelles 4 au niveau de la région pinéale ayant causé le décès (4 pour 16 BST d'une tumeur pinéale). Dans 2 cas le saignement s'est arrêté spontanément. Un patient a présenté une aggravation de l'état de conscience par la survenue d'un hématome sous dural qui fut opéré en urgence avec une bonne évolution post opératoire.

Depuis ces incidents, la stratégie thérapeutique ainsi que les modalités techniques de la BST ont été modifiées pour les tumeurs pinéales. En effet, une angio-IRM et une angiographie avec des temps phlébographiques ont été systématiquement réalisées pour ces localisations, afin de visualiser le trajet des veines cérébrales internes, qui coiffent généralement la tumeur, et qui font que toute biopsie par voie frontale est hasardeuse. Le point d'entrée et la trajectoire de la sonde ont été réalisés par voie orthogonale trans-temporale. Depuis lors, aucun incident hémorragique n'a été rapporté. En cas de processus pinéal très vascularisé, la BST est abolie au profit d'une biopsie à ciel ouvert qui permet de contrôler un éventuel saignement per-opératoire.

Trois autres décès sont survenus suite à une BST chez des patients dont le score de Glasgow préopératoire était $<$ ou $=$ à 7 . Depuis cet incident, la BST a été abolie chez des patients comateux. 
L'IRM permet la localisation de la lésion dans les trois plans de l'espace, ainsi que le calcul de la trajectoire, et de l'angle dans les plans sagittal et coronal. Elle a été réalisée dans 23 cas. Dans tous ces cas, elle fut concluante sans aucune complication. Aucun décès n'est survenu lors d'une BST en conditions IRM.

Le taux de mortalité global dans la série était de $2.7 \%$. Ce taux est passé de $5.9 \%(7 / 119)$, au cours de la première période (1995 à 2000), à $0 \%(0 / 137)$ au cours de la deuxième période (2000 à 2008) (Chi-carré; $p=0,013)$.

\section{DISCUSSION}

Le principal intérêt de la biopsie stéréotaxique est d'obtenir un diagnostic histologique dès lors qu'un abord chirurgical classique est jugé hautement risqué. II se conçoit que ces situations sont fréquentes dans la pratique de la neurochirurgie. II est également important de peser les indications en fonction de la difficulté technique de la biopsie, et des possibilités matérielles dont on peut disposer.

\section{Cadres stéréotaxiques}

Plusieurs types de cadres stéréotaxiques peuvent être utilisés : cadres de Leksell [13], de Brown Roberts Well (BRW) [4], Komai, ou de Radionics (CRW), principalement. Ces systèmes sont de maniement très simple et peuvent être utilisés en pratique quotidienne, même dans des centres peu spécialisés. Le cadre de Talairach [27] se prête bien à la réalisation d'artériographies stéréotaxiques, mais son utilisation au scanner est plus compliquée, nécessitant habituellement un cadre complémentaire [19].

\section{Choix du site de biopsie}

Les travaux de Kelly [11] et Daumas-Duport [6] ont bien démontré que les zones pathologiques prenant le contraste correspondaient pratiquement toujours à du tissu tumoral $(84.2 \%$ des cas). Les zones hypodenses apparaissent moins homogènes ; elles correspondent dans $75.5 \%$ des cas à du parenchyme cérébral infiltré de cellules tumorales isolées, dans $12.7 \%$ des cas à de la nécrose ou à de l'oedème et dans $11.8 \%$ des cas seulement à du tissu tumoral proprement dit.

Sur 184 prélèvements biopsiques, Greene [8] retrouve que les hypodensités centrotumorales (61 prélèvements) correspondent à du tissu tumoral dans $55.7 \%$ des cas et à de la nécrose dans 21.3 $\%$ des cas. Lorsque le prélèvement intéresse la prise de contraste périphérique, le prélèvement correspond dans $67.3 \%$ des cas à du tissu tumoral et dans $3.9 \%$ des cas à de la nécrose.

En cas de lésion de densité homogène (sans ou après injection de contraste), c'est le centre de la lésion qui constitue habituellement la cible. En cas de lésion à centre hypodense (kystique ou nécrotique), il est préférable de choisir une cible complémentaire en périphérie de la lésion, au niveau de la prise de contraste [18].

La biopsie proprement dite est habituellement réalisée au bloc opératoire. En l'absence de contrôle artériographique, certains auteurs préfèrent réaliser un trou de trépan classique de $10 \mathrm{~mm}$ de diamètre, afin d'ouvrir la dure-mère et ponctionner le cortex sous contrôle de la vue, limitant les risques d'hémorragie superficielle [18]. Pour notre part, nous jugeons qu'une simple trépanation percutanée (orifice de 2 à $3 \mathrm{~mm}$ ) peut être envisagée pour un abord frontal car le réseau veineux superficiel est relativement pauvre à ce niveau.

D'ailleurs, le taux d'hémorragie par lésion corticale est faible (1 hématome sous-dural aigu sur 283 cas). Cette trépanation peut donc être réalisée avec une relative sécurité.

Cas particuliers des tumeurs du tronc cérébral, de la région pinéale et du Ille ventricule En fonction de leur localisation exacte, les tumeurs du tronc cérébral peuvent être abordées par plusieurs voies. Les lésions du pédoncule cérébral ainsi que les lésions situées à la partie médiane de la protubérance, voire certaines lésions bulbaires peuvent être biopsiées par une voie frontale, suivant le grand axe du tronc cérébral $[18,10]$. Pour minimiser les lésions séquellaires liées à la 
traversée du tronc cérébral, il est recommandé d'utiliser des trocarts de faible diamètre $(1,5 \mathrm{~mm}$ de diamètre pour Hood [10]. Les lésions latérales du tronc cérébral ou les lésions des hémisphères cérébelleux sont habituellement abordées par voie transcérébelleuse sous-occipitale [18].

Les tumeurs de la région pinéale peuvent être abordées par voie orthogonale latérale ou en double obliquité (antérieure ou postérieure) [22].

La fréquence des complications liées à la biopsie des tumeurs de cette région varie selon les séries [3, 21, 22]. La mortalité $(1.3 \%)$ et la morbidité $(8 \%)$ de la stéréotaxie dans la région pinéale sont identiques à celles du reste de l'encéphale [9]. Cette morbidité relativement élevée est transitoire dans la moitié des cas et il n'y a que $0.8 \%$ de morbidité sévère $[12,14,15]$. Par ailleurs, il est reproché à la biopsie stéréotaxique de ne pas être représentative, surtout dans les tumeurs mixtes à composantes multiples pour lesquelles le pronostic et la conduite à tenir peuvent être tout à fait différents [5]. En fait, on peut considérer que la biopsie stéréotaxique est indiquée dans les cas où la clinique (surtout l'âge), l'imagerie et le dosage des marqueurs sont en faveur d'une tumeur radio- ou chimiosensible, ou dans les cas de tumeurs invasives ou disséminées [16].

Les kystes colloïdes du Ille ventricule sont habituellement abordés par une voie frontale [1, 17, 23, 24]. La trajectoire est calculée pour passer à travers le trou de Monro, en évitant les veines cérébrales internes et thalamo-striées. Une évacuation, même partielle, peut suffire à rétablir la circulation du liquide céphalorachidien et représenter une méthode thérapeutique définitive.

\section{Trocarts de biopsie et examen histologique}

Le trocart de biopsie le plus utilisé actuellement est le trocart aspiratif de Sedan [26]. Son diamètre externe est de $2.5 \mathrm{~mm}$. II permet d'effectuer des prélèvements cylindriques de $1.5 \mathrm{~mm}$ de diamètre et de 5 à $10 \mathrm{~mm}$ de long. Pour augmenter la fiabilité de la biopsie, il est recommandé d'effectuer plusieurs prélèvements. Ceci peut être réalisé en effectuant plusieurs prélèvements au niveau du même site de biopsie en faisant simplement tourner le trocart sur lui-même à chaque fois (prélèvement en " rosette "). Les prélèvements peuvent être étagés le long de la trajectoire, en laissant un intervalle de $2 \mathrm{~mm}$ entre chaque site de biopsie [18].

\section{IRM}

Le cadre Radionics (CRW), à l'instar de la plupart des cadres récents, est compatible avec ce type d'imagerie, et les systèmes de repérage sont également adaptés à tous les types d'antennes IRM. Le repérage IRM est particulièrement utile lorsque les limites tumorales sont mal définies en scanographie [28]. La meilleure visualisation des structures anatomiques - notamment vasculaires - par rapport au scanner, est également un élément important pour le choix de certaines voies d'abord difficiles [7]. II ne faut cependant pas sous-estimer les possibilités d'erreurs liées au repérage IRM. L'interprétation des zones réellement tumorales n'est pas encore toujours évidente et les erreurs de mesure liées à la déformation des images par le champ magnétique existent encore $[12,25]$. Dans notre étude, l'IRM a réduit le taux de biopsies blanches et a diminué les risques de complications hémorragiques.

Etude des principales séries de la littérature La série française publiée en 1985 [2], représente la plus grande série de biopsies stéréotaxiques retrouvée dans la littérature (11 centres, 3052 cas). La biopsie s'est révélée négative (cible manquée) dans 1 à $15 \%$ des cas, imprécise (résultat faux ou incertain) dans 1 à $27 \%$ des cas. La corrélation entre le résultat histologique de la biopsie et celui de la pièce opératoire (en cas d'exérèse secondaire) s'est avérée bonne dans 60 à $93 \%$ des cas. La mortalité postopératoire a varié de 0 à $2.5 \%$ des cas $(0.65 \%$ en moyenne). La mortalité a pratiquement toujours été liée à un accident hémorragique. Une aggravation neurologique transitoire a été constatée dans $4.5 \%$ des cas ; des aggravations neurologiques permanentes sont apparues dans $1 \%$ des cas.

Sur 302 biopsies stéréotaxiques réalisées par Ostertag [20], la mortalité postopératoire a été de $2.3 \%$ des cas ; une aggravation neurologique transitoire a été observée dans $3 \%$ des cas.

Daumas-Duport [6] insiste sur l'intérêt des biopsies étagées qui permettent de préciser la configuration spatiale des tumeurs.

Chandrasoma [5] a rapporté une série de 30 patients ayant eu une exérèse chirurgicale après une biopsie stéréotaxique. II y a eu une parfaite corrélation entre les résultats histologiques dans 19 
cas sur 30 (63\% des cas). II y a eu 2 cas de véritable erreur de diagnostic (6,6\% des cas). Dans 9 cas, le diagnostic s'est avéré simplement imprécis.

\section{CONCLUSION}

L'amélioration de la stratégie et de la procédure de la BST a rendu cette technique sûre et efficace pour le diagnostic des tumeurs cérébrales même en cas de tumeur pinéale ou du tronc cérébral. Afin d'améliorer l'efficacité, les auteurs recommandent de réaliser des biopsies étagées, de multiplier les BST en cas d'histologie non concluante et de réaliser le guidage en conditions IRM pour les petites lésions profondes. Les lésions pinéales nécessitent une étude précise de leur vascularisation par IRM et angiographie avec des temps phlébographiques. La BST de ces tumeurs doit être réalisée par voie orthogonale trans-temporale. Pour cette localisation, la voie frontale antérieure est proscrite.

Tableau 1 : Localisation des tumeurs cérébrales

\begin{tabular}{|l|c|c|}
\hline \multicolumn{1}{|c|}{ Localisation } & Nombre & Pourcentage \\
\hline Lobaire & 160 & $56.5 \%$ \\
\hline Diencéphalique & 56 & $20 \%$ \\
\hline Multiple & 40 & $14 \%$ \\
\hline Pinéale & 17 & $6 \%$ \\
\hline Tronc cérébral & 7 & $2.5 \%$ \\
\hline Ventriculaire & 3 & $1 \%$ \\
\hline Total & $\mathbf{2 8 3}$ & $\mathbf{1 0 0} \%$ \\
\hline
\end{tabular}

Tableau 2 : Résultats histopathologiques

\begin{tabular}{|l|c|c|}
\hline \multicolumn{1}{|c|}{ Type histologique } & Nombre & Pourcentage \\
\hline \multicolumn{1}{|c|}{ Gliomes } & $\mathbf{1 6 7}$ & $\mathbf{5 9} \%$ \\
\hline Astrocytomes & & $33.5 \%$ \\
\hline Bas grade & & $21.2 \%$ \\
\hline Haut grade & & $12.3 \%$ \\
\hline Glioblastomes multiformes & & $18.7 \%$ \\
\hline Oligodendrogliomes & $\mathbf{4 0}$ & $6.8 \%$ \\
\hline Métastases & $\mathbf{3 3}$ & $\mathbf{1 4 . 2} \%$ \\
\hline Tuberculomes & $\mathbf{1 7}$ & $\mathbf{1 1 . 6} \%$ \\
\hline Lymphomes & $\mathbf{8}$ & $\mathbf{2 . 8} \%$ \\
\hline Tumeurs germinales & $\mathbf{9}$ & $\mathbf{3 . 2} \%$ \\
\hline Tumeurs du parenchyme pinéal & $\mathbf{9}$ & $\mathbf{3 . 2 \%}$ \\
\hline Autres & $\mathbf{2 8 3}$ & $\mathbf{1 0 0 \%}$ \\
\hline Total & &
\end{tabular}




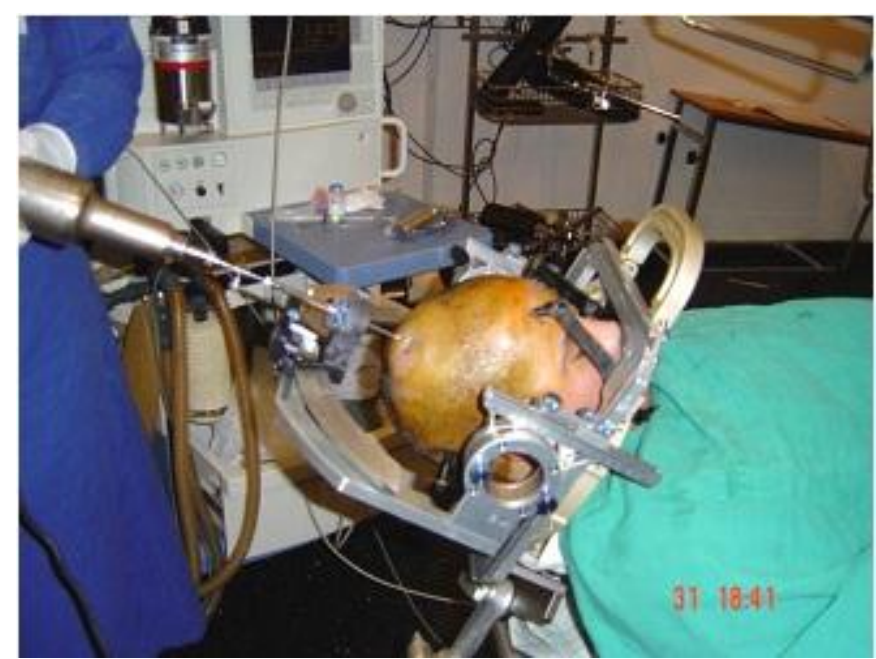

Figure 1:

Réalisation d'un trou de trépan en condition stéréotaxique

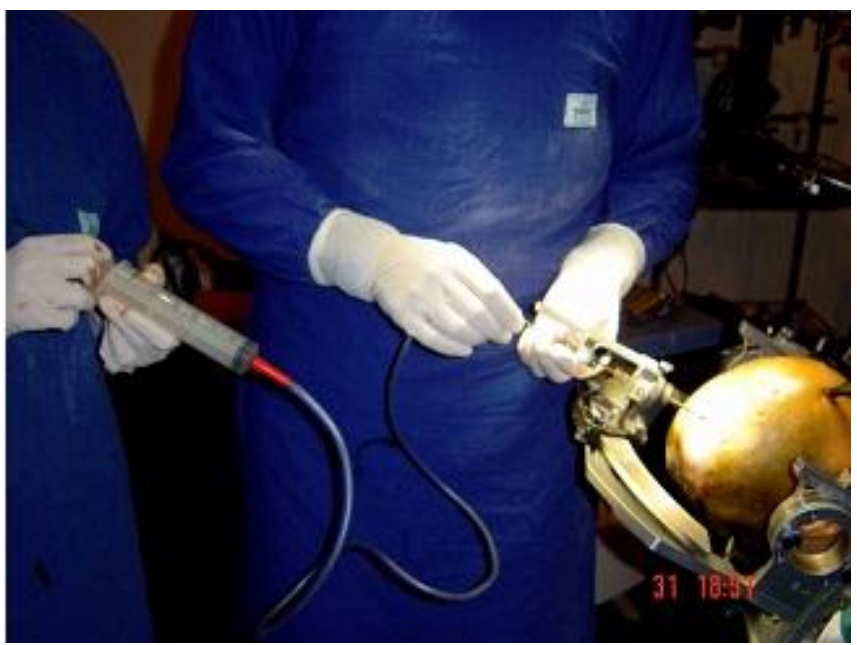

Figure 2:

Prélèvement de l'échantillon tumoral en condition stéréotaxique 


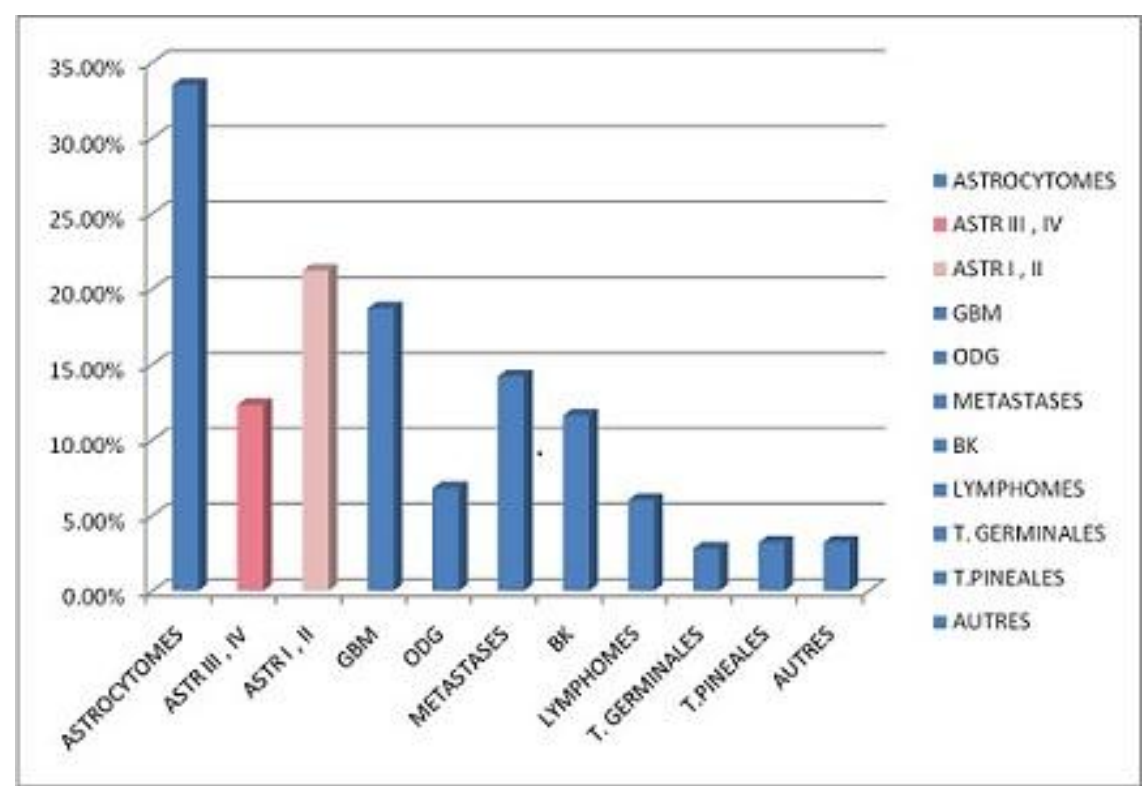

Figure 3 :

Résultats histopathologiques. 


\section{REFERENCES}

1. ABERNATHEY CD, DAVIS DH, KELLY PJ Treatment of coloid cysts of the third ventricle by stereotactic microsurgical laser craniotomy. J Neurosurg 1989; 70: 524-529

2. BENABID A, BLOND S, CHAZAL $J$ coll. Les biopsies stéréotaxiques des néoformations intracrâniennes. Réflexions à propos de 3052 cas Neurochirurgie 1985 ; 31: 295-301

3. BOSCH DA Indications for stereotactic biopsy in brain tumors. Acta Neurochir 1980; 54: 167-179

4. BROWN RA, ROBERTS TS, OSBORN AG Simplified CT-guided stereotactic biopsy. AJNR 1981; 2: 181-184

5. CHANDRASOMA PT, SMITH MM, APUZZO ML Stereotactic biopsy in the diagnosis of brain masses: Comparison of results of biopsy and resected surgical specimen. Neurosurgery 1989; 24: 160-165

6. DAUMAS-DUPORT C, MEDER JF, MONSAINGEON $V$ coll. Les gliomes cérébraux : Malignité, délimitation et configuration spatiale. Données comparatives, biopsies cérébrales étagées stéréotaxiques-tomodensitométrie (étude préliminaire à propos de 50 cas) J Neuroradiol 1983; 10: 5180

7. EHRICKE HH, SCHAD LR, GADEMANN G, et al. Use of MR angiography for stereotactic planning. J Comput Assist Tomogr 1992; 16: 35-40

8. GREENE GM, HICHON PW, SCHELPER RL, et al. Diagnostic yield in CT-guided stereotactic biopsy of gliomas. J Neurosurg 1989; 71: 494-497

9. HAYEK G, FOURNIER D ET GUY G. Tumeurs de la région pinéale. Encycl Méd Chir (Editions Scientifiques et Médicales Elsevier SAS, Paris, tous droits réservés), Neurologie,17-380-D-10, 2002, 9 $\mathrm{p}$

10. HOOD TW, GEBARSKI SS, McKEEVER PE, VENES JL Stereotaxic biopsy of intrinsec lesions of the brain stem. J Neurosurg 1986; 65: 172-176

11. KELLY PJ, DAUMAS-DUPORT C, KISPERT DB, et al. Imaging-based stereotaxic serial biopsies in untreated intracranial glial neoplasms. J Neurosurg 1987; $66: 865-874$

12. KONDZIOLKA D, DEMPSEY PK, LUNDSFORD LD , et al. A comparison between magnetic resonance imaging and computed tomography for stereotactic coordinate determination. Neurosurgery 1992; 3 : 402-407

13. LEAKSELL L, JERNBERG B Stereotaxis and tomography. A technical note Acta Neurochir $1980 ; 52$ $: 1-7$

14. MORANTZ RA Radiation therapy in the treatment of cerebral astrocytoma. Neurosurgery 1987; 20 : 975-982

15. MRC Working Party on Misonidazole in gliomas A study of the effect of Misonidazole in conjunction with radiotherapy for the treatment of grade 3 and 4 astrocytoma. Br J Radiol 198; 56: 673-682

16. MULLER PJ, WILSON BC Photodynamic therapy of malignant primary brain tumors: clinical effects, post-operative ICP and light penetration of the brain. Photochim Photobiol 1987; 46: 929-935

17. MUSOLINO A, MUNARI C, BLOND S coll. Diagnostic et traitement stéréotaxique des kystes colloïdes du troisième ventricule. A propos de 7 cas Neurochirurgie 1988; 34: 26-36

18. NGUYEN JP, Decq $P$, Kéravel $Y$, Approche stéréotaxique, diagnostique et thérapeutique des tumeurs cérébrales-Editions techniques-Encycl Méd Chir (Paris, France), Neurologie, 17-210-A-310,1995.

19. NGUYEN JP, SZIKLA G, MISSIR O Flabilité d'une méthode simplifiée de transposition des images tomodensitométriques. Corrélations au repérage stéréotaxique dans 30 cas Neurochirurgie 1982; 28: 271-274

20. OSTERTAG CB, MENNEL HD, KIESLING M Stereotactic biopsy of brain tumors. Surg Neurol 1980; 14: $275-283$

21. PECKER J, SCARABIN JM, VALLEE B, coll.Treatment in tumors of the pineal region: value of stereotactic biopsy. Surg Neurol $1979 ; 12: 341-348$

22. PERAGUT JC, DUPARD T, GRAZIANI N, SEDAN R De la prévention des risques de la biopsie stéréotaxique de certaines tumeurs de la région pinéale : à propos de 3 observations. Neurochirurgie 1987; 33: 23-27

23. PERAGUT JC, RISS JM, FARNARIER P coll. Kystes colloïdes du 3e ventricule. Scanner, IRM et ponction stéréotaxique. A propos de 9 observations Neurochirurgie 1990; 36: 122-128

24. RIVAS JJ, LOBATO RD CT-assisted stereotaxic aspiration of colloid cysts of the third ventricle. J Neurosurg 1985; 62: 238-242

25. SCHAD L, LOTT S, SCHMITT F, et al. Correction of spatial distorsion in MR imaging: A prerequisite for 
accurate stereotaxy. J Comput Assist Tomogr 1987; 11: 499-505

26. SEDAN R, PERAGUT JC, FARNARIER P coll. Place de la biopsie en condition stéréotaxique dans la tactique des gliomes malins. Neurochirurgie 1981; 27: 285-286

27. TALAIRACH J, PERAGUT JC, FARNARIER Ph, MANRIQUE M. The role of the stereotactic radiographic exploration in neurosurgical interventions. In: Salamon $G$ ed. Advances in cerebral angiography. Springer Verlag. Berlin. 1975

28. TARATUTO AL, SEVLEVER G, PICCARDO P Clues and pitfalls in stereotactic biopsy of the central nervous system. Arch Pathol Lab Med 1991; 115: 596-602 